\title{
ON THE PREDICTIVE POWER OF THE MINIMUM ENERGY CONDITION
}

\author{
MARTIN POHL \\ Max-Planck-Institut für Radioastronomie, Auf dem Hügel 69, W-5300 Bonn 1, FRG
}

November 2, 1992

\begin{abstract}
We reexamine the minimum energy method to determine the magnetic field strength in spiral galaxies from the cosmic ray standpoint of view. It is shown that for example in M51 the estimated field strength is about a factor of 2 lower than obtained with the standard method. As a by-product the corresponding $\gamma$-ray flux from the galaxies can be calculated, which will allow further improvement of the method provided reliable $\gamma$-ray spectra are at hand.
\end{abstract}

\section{The method}

Since the minimum energy method employs a minimization of the energy density sum of cosmic rays and magnetic field, the crucial point in it is the extrapolation from the radio flux at a given frequency to the energy density in cosmic rays, which is commonly done by a series of secondary assumptions. We calculate the steady-state number and energy densities for cosmic ray protons and electrons under the standard assumption of power-law injection in momentum. The asymptotic formulae allow us to base the magnetic field estimate by the minimum energy condition on a more physical ground relaxing on ad-hoc assumptions like a ratio of 100 between the energy densities in protons and electrons.

The principal procedure is as follows: we start with a power-law injection rate for protons and electrons.

$$
Q_{e, p}=q_{e, p}\left(m_{e, p} c\right)^{s-1} p^{-s}
$$

After changing to the particle's kinetic energy as variable the integrated particle spectra in a thick target can be calculated as

$$
N_{e, p}(E)=|\dot{E}|_{e, p}^{-1} \int_{E} d u Q_{e, p}(u)
$$

where $\dot{E}$ denotes all relevant energy loss terms. These integrated particle spectra reveal spectral breaks caused by different energy dependences of the loss mechanisms. By observing such a break in the integrated radio spectrum we obtain the injection spectral index $s$, and a relation between the gas density and the magnetic field strength.

The number density of particles can be calculated via the equivalent confinement volume (see Jones 1991), for which we assume that diffusion is the dominant transport process.

$$
N(E, x \in V(E))=N(E) V^{-1}(E), \quad \text { where } \quad V(E) \simeq 4 \pi R^{2} \sqrt{D E|\dot{E}|^{-1}}
$$


where $R$ is the equivalent galactocentric radius and $D$ is the diffusion coefficient. Investigations for solar cosmic rays and the secondary to primary ratio in galactic cosmic rays indicate that the mean free path follows a power-law in rigidity.

$$
D=\frac{v}{3} \lambda=D_{0} v(p c)^{a}
$$

Knowledge of a spectral break in the integrated radio spectrum, i.e. the relation between the gas density and the magnetic field strength, now allows us to write the energy density of cosmic rays as a function of the radio flux at a given frequency and the magnetic field strength, and the minimization of the energy sum can be performed.

From the relation between gas density and magnetic field strength we infer as a by-product without further assumptions an estimate for the mean gas density which determines the $\gamma$-flux due to $\pi^{0}$-decay and non-thermal bremsstrahlung. Please note, that part of the electron flux might be secondary in origin, since inevitably in a thick target in the hadronic interactions of the protons $\pi^{ \pm}$-mesons are produced which decay to $e^{ \pm}$.

\section{The example: M51}

From the integrated radio spectrum of M51 we find a spectral break centered on $1.4 \mathrm{GHz}$ (Pohl 1992). As long as reliable $\gamma$-ray spectra are not available for this galaxy, we cannot measure the flux ratio between protons and electrons. Thus we need to assume that both were injected with the same spectral index $s$ and the same total injection rate (Bell 1978a,b). Then in M51 the magnetic field strength can be estimated to be $B \simeq 6.5 \mu G$. The mean gas density can be given in turn. This value for the magnetic field strength is about a factor of 2 lower than previous findings based on the standard method of relying on the injection spectra instead of the equilibrium spectra. The discrepancy is mainly due to two assumptions made in earlier papers on this topic.

Firstly, we relax on a prescibed ratio between the energy densities in protons and electrons. Furthermore, we employ the full spectral shape of the electron spectrum and not extraploations from a small part of it to determine the energy density in electrons.

The validity of the method presented here can be further tested by observations in the $\gamma$ - and neutrino-regime.

\section{References}

Bell, A.R.: 1978a, MNRAS 182, 147

Bell, A.R.: 1978b, MNRAS 182, 443

Jones, F.C.: 1991, Proceedings IAU 144, ed. J.G.B.M.Bloemen, Kluwer, p.359

Pohl, M.: 1992, $A A$, submitted 